\title{
Port, Aménagement et Développement Durable À San-Pedro (Sud-ouest de la Côte d'Ivoire)
}

\author{
Ogou Atsé Willy Arnaud, Doctorant \\ Jean Tapé Bidi, Professeur titulaire
}

Université Félix Houphouët-Boigny Abidjan, Côte d'Ivoire

URL:http://dx.doi.org/10.19044/esj.2019.v15n8p110

\section{Résumé}

Pour accroître le trafic portuaire et répondre efficacement aux attentes des opérateurs économiques, d'importants travaux d'aménagement ont été réalisés au port de San-Pedro et dans son arrière-pays. Les aménagements effectués induisent des interrogations sur les rapports entre ces multiples investissements et la question du développement durable à San-Pedro. Avec l'insistance sur les questions environnementales à l'échelle planétaire, la tendance actuelle est à l'intégration des principes du développement durable dans toutes les activités économiques. La présente étude a pour objectif d'étudier l'impact des aménagements réalisés dans la région Sud-ouest dont l'ouverture du port sur les composantes du développement durable. La méthode de travail déployée s'est appuyée sur la recherche documentaire, et l'enquête de terrain. Les investigations révèlent que les aménagements portuaires ont des retombées économiques et sociales indéniables pour la ville de San Pedro. Mais, ils sont à l'origine de problèmes environnementaux notamment la dégradation des ressources forestières, les pollutions diverses, l'érosion côtière, etc. Ces problèmes compromettent la possibilité des générations futures d'avoir un environnement sain et stable.

Mots clés : San-Pedro, aménagement portuaire, développement durable, environnement, impact, 


\title{
Port, Planning and Sustainable Development in San- Pedro (Southwest of Ivory Coast)
}

\author{
Ogou Atsé Willy Arnaud, Doctorant \\ Jean Tapé Bidi, Professeur titulaire
}

Université Félix Houphouët-Boigny Abidjan, Côte d’Ivoire

\begin{abstract}
Important works have been done on San-Pedro PORT and its hinterland in order to meet the expectations of investors but also to intensify trafficking. The work accomplished implies questions on the relations existing between those investments and the sustainable development matter in SanPedro. Considering the stress put on environmental matters at a global scale, it is a must to fit into the requirements of sustainable development in all of the economic activities implemented. The actual study aims at studying the impact of the work accomplished in the southwest one of which is the shifting of the port to the elements of sustainable development. The methodology of the study was based on research, on surveys. The surveys revealed that the work done on the port has undeniably good both at the social but economical level for the town of San Pedro. However, its side effects are visible on the environment, for instance, the destruction of the environment, pollution, coastal erosion... These problems prevent the future generations of benefiting of a pure and liveable environment.
\end{abstract}

Keywords: San-Pedro, work, sustainable development, environment, impact

\section{INTRODUCTION}

Le développement durable est un terme très en vogue. Il est défini comme un développement qui répond aux besoins du présent sans compromettre la capacité des générations futures de répondre aux leurs (rapport Brundtland, 1987, p. 40). Selon ce rapport, le développement durable a pour objectif principal de concilier le progrès économique et social avec la préservation de l'environnement. Le dernier volet de ce développement, à savoir l'environnement, doit être intégré à toutes les activités économiques. À ce titre, le déroulement des activités maritimes et portuaires doit nécessairement s'inscrire dans une logique de développement durable. Avec le développement de l'économie portuaire et la recherche des 
profits, il apparaît important de se demander la place accordée à la préservation des écosystèmes naturels pour le futur des territoires et générations à venir.

Située dans le Sud-ouest de la Côte d’Ivoire (figure 1), la ville de SanPedro et son port offre un cadre favorable pour l'étude des relations entre aménagement portuaire et développement durable. La ville de San-Pedro et son port ont fait l'objet d'une multitude d'études. On peut citer entre autres, celles de Tapé (2004), Kablan (2008), Loba (2010), Kassi (2012), et N'guessan (2010, 2013 ; 2014 et 2017). Ces études ont mis l'accent sur le rôle du port de San-Pedro dans l'urbanisation de ladite ville. Elles donnent également des informations sur l'importance des aménagements portuaires dans la compétitivité du port de San-Pedro et mettent en évidence les effets néfastes des activités portuaires sur l'environnement. Malgré cette abondance littéraire, très peu d'informations existent sur les liens entre les aménagements portuaires et le développement durable de San-Pedro. Pourtant, cette ville en raison de son port constitue un terrain propice pour étudier les relations entre les aménagements portuaires et la question du développement durable. Avec l'ouverture du port, la ville de San-Pedro et toute la région du Sud-ouest ont bénéficié de divers travaux d'aménagement. Ces investissements ont joué un rôle majeur dans le processus de diversification du trafic du port de San-Pedro et permis à ce dernier d'enregistrer des trafics importants. Avec l'importance prise par l'activité maritime à San-Pedro, on assiste au phénomène de littoralisation. Or, les littoraux sont reconnus comme des espaces fragiles (Choblet 2005, p. 51). Dès lors, le foisonnement des activités humaines sur le littoral de San-Pedro rend urgente une réflexion scientifique sur la pérennité des activités économiques induites par la présence du port et le futur de ladite ville.

La présente étude se propose donc de mettre en évidence les aménagements opérés pour le développement du port et de la ville de SanPedro en analysant leur impact sur le développement durable.

\section{MÉTHODOLOGIE}

La recherche documentaire et l'enquête de terrain ont été les principales méthodes de collecte des données. La recherche documentaire nous a conduit sur internet, dans des bibliothèques et les centres de documentation. Les documents consultés concernent des thèses, mémoires, articles, des rapports annuels de l'Autorité pour l'Aménagement de la Région du Sud-ouest (ARSO) et du port de San-Pedro. La littérature explorée met en exergue les relations entre le port et la ville, les rapports entre les aménagements portuaires et le développement durable de façon générale. Les informations tirées de la recherche documentaire ont été complétées avec celles issues des investigations de terrain. Les enquêtes se sont déroulées entre 2016 et 2018. À ce niveau, trois techniques ont été utilisées : l'observation, 
l'entretien et l'enquête par questionnaire. L'observation a consisté essentiellement à parcourir la ville de San-Pedro. Les principales entités observées sont les exploitations agricoles, les ressources naturelles (forêts, eau, ...), les industries, les activités économiques, les infrastructures et équipements. Cet exercice a permis d'une part de mieux appréhender la dynamique spatiale de l'espace urbain et d'autre part de s'imprégner du comportement des différents acteurs qui interviennent dans la vie économique de San-Pedro et leurs modes de gestion des ressources naturelles. Les entretiens ont été réalisés auprès de trois chefs de service du Port Autonome de San-Pedro (PASP), du Directeur régional des Eaux et Forêts du Bas Sassandra et du Directeur de la Société de Développement des Forêts (SODEFOR) de San-Pedro. Les échanges ont porté sur les effets des activités portuaires sur la ville de San-Pedro et l'état de l'environnement. L'enquête par questionnaire a eu pour cible les responsables des entreprises et les commerçants installés sur la place portuaire et en zone industrielle. Ce choix s'explique par le fait que le sujet s'intéresse spécifiquement à ces deux entités. La méthode empirique à choix raisonné a servi pour les enquêtes. Un questionnaire a été adressé à tous les opérateurs économiques exerçant sur la place portuaire. Mais le refus de certains acteurs n'a pas permis d'interroger toutes les personnes sollicitées. Le tableau 1 ci-après fait le récapitulatif des entreprises visitées et des commerçants enquêtées.

Tableau 1 : Répartition des entreprises et commerçants sollicitées

\begin{tabular}{|c|c|c|c|}
\hline Structures & $\begin{array}{c}\text { Nombre } \\
\text { d'entreprises } \\
\text { identifiées }\end{array}$ & $\begin{array}{c}\text { Effectif } \\
\text { interrogés }\end{array}$ & $\begin{array}{c}\text { Taux de } \\
\text { couverture en (\%) }\end{array}$ \\
\hline Industrie Agro-alimentaire & 23 & 17 & 73,91 \\
\hline Industrie de bois & 14 & 14 & 100 \\
\hline Société maritime & 14 & 10 & 71,42 \\
\hline Commerçants & 350 & 196 & 56 \\
\hline Total & 401 & 237 & \multicolumn{1}{|c}{} \\
\cline { 1 - 2 } & & &
\end{tabular}

Source : enquêtes de terrain, 2018

Les données recueillies ont été traitées à l'aide de l'outil informatique. Le logiciel Word a été utilisé pour le traitement du texte, Excel pour la réalisation des tableaux et graphiques. La confection des cartes a été possible par l'entremise du logiciel Arc Gis. 
Figure 1 : Localisation de la ville de San-Pedro dans le Sud-ouest ivoirien

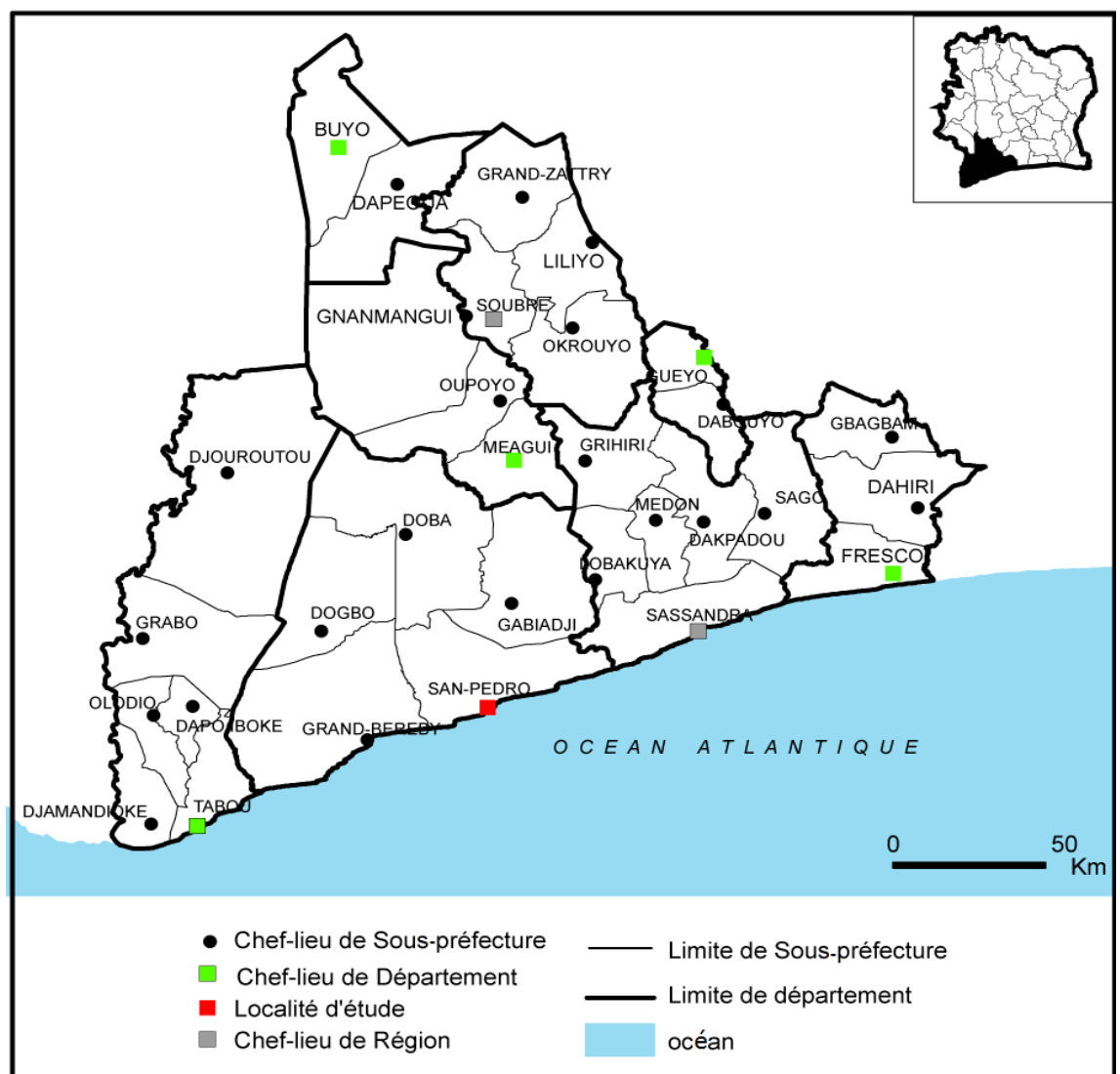

Source : BNETD, 2011

Réalisation: OGOU Atsé, 2019

\section{II-RÉSULATS}

Les informations obtenues ont permis d'articuler le travail en trois points. Le premier passe en revue les opérations de développement réalisées dans le Sud-ouest ivoirien avec l'ouverture du port. Le second étudie l'impact des aménagements sur le développement socio-économique de San-Pedro. Le troisième met en exergue les problèmes environnementaux induit par les activités portuaires.

\section{1-Les principaux aménagements réalisés dans le Sud-ouest ivoirien pour le développement du port}

Les actions posées pour accroître le trafic du port et favorisé le développement de San-Pedro et de toute la région du Sud-ouest ivoirien ont porté en milieu urbain sur la mise en place d'infrastructures routières. En zone rurale, les investissements ont reposé essentiellement sur l'aménagement d'espace agricole. 


\section{1-1- La création des plantations agro-industrielles et villageoises pour alimenter le trafic portuaire}

Un port, pour son fonctionnement a besoin de matières premières. En côte d'Ivoire, comme dans beaucoup de pays en voie développement, la politique adoptée par l'État pour promouvoir le développement du trafic du port de San-Pedro a reposé sur l'agriculture. Pour ce faire, un développement à grande échelle d'une agriculture industrielle et villageoise s'est opéré à SanPedro et son arrière-pays, un territoire peu peuplé où le facteur "terre" n’était pas limitant (Schwartz, 1993, p. 343). Quatre opérations agro-industrielles ont été engagées entre 1971 et 1975 : 2 opérations "hévéa”, 1 opération de "palmier à huile” et l'opération de "cocotier” (tableau 2).

Tableau 2 : Superficies des plantations industrielles et villageoises

\begin{tabular}{|l|c|c|l|c|}
\hline \multirow{2}{*}{ Opérations } & \multicolumn{3}{|c|}{ Superficies prévues } & \multicolumn{2}{l|}{$\begin{array}{l}\text { Superficies réalisées à la fin de } \\
\text { l'opération ARSO }\end{array}$} \\
& $\begin{array}{l}\text { Plantations } \\
\text { industrielles }\end{array}$ & $\begin{array}{l}\text { Plantations } \\
\text { villageoises }\end{array}$ & $\begin{array}{l}\text { Plantations } \\
\text { industrielles }\end{array}$ & $\begin{array}{l}\text { Plantations } \\
\text { villageoises }\end{array}$ \\
\hline Hévéa à grand- Béréby & 13500 ha & & 13525 ha & \\
\hline Hévéa à Rapides-Grah & 5000 ha & & 5245 ha & \\
\hline Palmier à Tabou & 11000 ha & 2000 ha & 7087 ha & 42 ha \\
\hline Cocotier à Tabou & 7500 ha & 2000 ha & 6300 ha & 220 ha \\
\hline
\end{tabular}

Source: SRAT, 2008

La première opération "hévéa” est lancée dans la Sous-préfecture de Grand Béréby, en pays Krou avec pour objectif la réalisation d'une plantation industrielle de 13500 ha. La seconde opération “hévéa” est entreprise en 1974 dans la forêt dite de Rapides-Grah, à quelque $80 \mathrm{~km}$ au Nord de San-Pedro, en pays Bakwé avec pour objectif la réalisation d'une plantation industrielle de 5000 ha. Les opérations "palmier à huile et cocotier" démarrent conjointement dans la Sous-préfecture de Tabou, en pays krou en 1975. En ce qui concerne le palmier, l'objectif est la réalisation de 11000 ha de plantations industrielles et de 2000 ha de plantations villageoises. Quant au cocotier, il consiste en la création de 7500 ha de plantations industrielles et de 2000 ha de plantations villageoises. La gestion est confiée à la Société pour le Développement du Palmier à Huile (SODEPALM). Finalement, sur les 7500 ha de plantations industrielles prévu pour le cocotier à Tabou, c'est 6300 ha qui ont été créés. Les prévisions non atteintes au niveau des plantations industrielles se justifient par le manque de financement dû à la crise économique de 1980 qui a frappé la Côte d'Ivoire. Cette période peu favorable à de gros investissements a contraint l'État ivoirien à arrêter les projets prévus. Pour ce qui est des plantations villageoises, les résultats souhaités n’ont pas été atteint à cause de la faible adhésion des kroumen aux différents projets agricoles. L'économie du Sud-ouest ivoirien est orientée vers l'activité 
maritime. Elle constituait la principale activité génératrice de revenu. Selon Schwartz (1993, p. 310), ni la culture de rente, ni l'exploitation forestière, ni l'activité industrielle ne sont vraiment créatrices de revenu pour les populations autochtones.

À côté des cultures susmentionnées, les cultures de cacaoyer et caféier ont été fortement encouragées par l'État. La promotion des cultures cacaoyères et caféières est confiée à la Société d'Assistance Technique pour la Modernisation de l'Agriculture en Côte d'Ivoire (SATMACI). Cette structure était chargée du conseil agricole, de la diffusion du matériel végétal et autres intrants y compris des crédits aux agriculteurs. De 1973 à 1979 plus de 2000 ha de plantations individuelles de cacao et 1500 ha de café sont réalisées dans la Sous-préfecture de San- Pedro avec l'aide de la SATMACI (Schwartz, 1993, p. 363).

Les productions issues de l'arrière-pays national sont convoyées vers la ville de San-Pedro où elles sont conditionnées et manutentionnées puis exportées via le port.

\section{1-2-L’ouverture des voies pour l'acheminement des marchandises vers le port}

La route occupe une place importante dans le transport des marchandises et des personnes. D’elle dépende la qualité et la rapidité dans l'acheminement des produits des zones de production vers les zones de grande consommation. Cependant, l'état du réseau routier du Sud-ouest ne favorise pas l'acheminement rapide des produits agricoles des zones de production. Schwartz (1993, p. 339) indique que l'infrastructure routière jusqu'à la fin des années soixante se composait principalement de pistes d'exploitation et de pistes de liaisons réalisées de façon sommaire par les exploitants forestiers. Pour pallier cette situation, un important réseau d'infrastructures routières est mis en place entre 1969 et 1981. Le réseau routier avait pour principal objectif de faciliter le transit des produits agricoles du Sud-ouest vers le port de SanPedro. Le bilan routier entre 1969 et 1981 se présente comme suit : les routes bitumées passent de $15 \mathrm{~km}$ à $228 \mathrm{~km}$ et les routes en terre et les pistes de 1 $579 \mathrm{~km}$ à $3107 \mathrm{~km}$ (Hauhouot, 2002, p. 209). L'amélioration des voies terrestres apporte une part importante dans le processus de désenclavement de la région et l'évacuation des produits de l'hinterland vers le port.

\section{2-Impact des activités portuaires sur le développement de San-Pedro}

La présence du port a fait de San-Pedro un lieu de prédilection innombrables activités économiques. Celles-ci ont apporté des transformations indéniables dans la ville de San- Pedro. Aussi faut-il le rappeler qu'elles procurent des revenus substantiels aux populations. 


\section{2-1-Le port, comme facteur d'extension spatiale de la ville}

Jadis, hameau d'une quarantaine d'habitants San-Pedro depuis l'ouverture des chantiers du projet ARSO connaît une croissance démographique remarquable. Selon Loba (2010, p. 8), la population a été multipliée par 9 sur la période allant de 1969 à 1975. Elle est passée de 3800 habitants à plus de 30 000. Le même auteur indique qu'entre 1972 et 1973, la ville a vu sa population passer du simple au double de 13419 à 27550 habitants. Cette explosion démographique est en grande partie liée à l'ouverture du port. En effet, cette immense infrastructure a transformé SanPedro en un pôle d'attraction. À cette époque, la ville et son port apparaissaient aux yeux des populations comme un eldorado, tant les activités étaient florissantes. Cette situation a conduit d'une part à plusieurs vagues migratoires de ressortissants de la sous-région ouest africaine, et d'autre part des populations issues des autres régions de la Côte d'Ivoire. Ces mouvements migratoires se sont poursuivis et accentués à partir des années 80 avec le déploiement des unités agro-industrielles et des activités connexes qui nécessitaient une main d'œuvre abondante. Les données tirées des recensements de la population réalisés par l'Institut National de Statistique (INS) en 1975, 1988 et 1998 indiquent que la ville de San-Pedro enregistre des taux d'accroissement annuel de 6,5\% entre 1975 et 1988, et 6,1 \% entre 19881998.

La pression démographique inhérente à la dynamique des activités portuaires s'est suivie de besoins en logements. Cela a fortement contribué à l'accroissement des surfaces bâties et par ricochet à la croissance spatiale de la ville de San-Pedro. Pour mieux apprécier la dynamique spatiale de SanPedro, l'analyse s'appuie sur les données de la figure 2.

Figure 2 : Accroissement de la surface urbanisée à San-Pedro

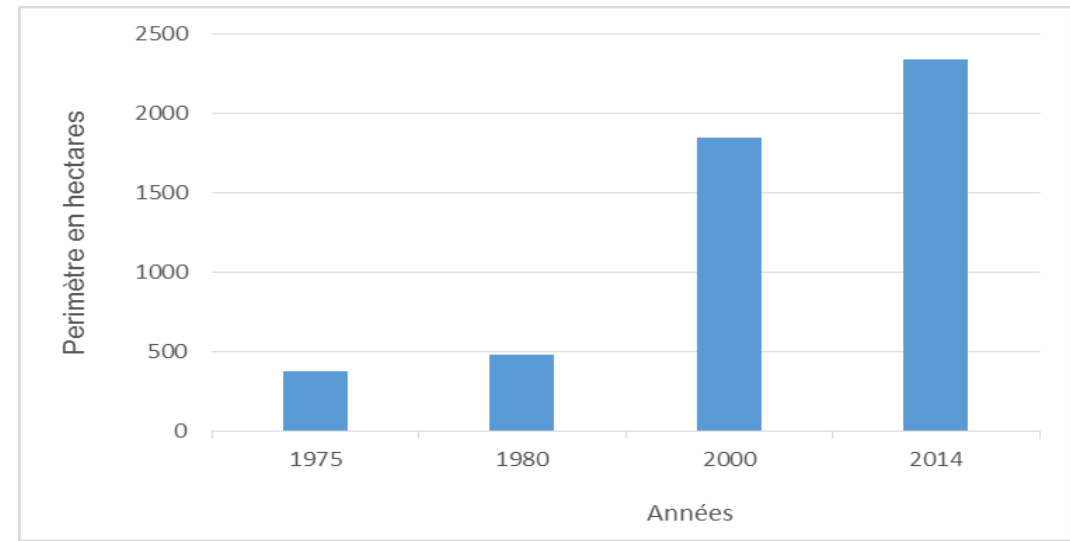

Source : RPG, 1975, INS : Estimation 1980 et 2000 et MEMPD², 2015

${ }^{2}$ MEMPD : Ministère d’État, Ministère du plan et du développement 
Selon les données de la figure 2, la ville est établie au départ sur une plate-forme aménagée d'environ 306 ha. En 1975, la superficie urbanisée atteint 335 ha, puis 480 ha en 1980 et 1850 ha en 2000. En 2014, l'espace urbanisé est passé à 2340 ha. Le processus de l'étalement urbain s'est fait à travers les différents lotissements mis en place par l'ARSO et le conseil municipal.

\section{2-2-Une présence remarquable d'entreprises aux retombées socio- économiques}

La zone portuaire de San-Pedro est animée par une panoplie d'activités. On y trouve de nombreuses entreprises. Celles-ci opèrent principalement dans la transformation du bois, le transport maritime et l'agroalimentaire.

Dans leur fonctionnement, les entreprises versent des sommes importantes à la mairie. L'enquête de terrain n'a pas permis de collecter les statistiques sur les taxes versées par les entreprises. Ces informations selon la marie et les sociétés concernées par nos enquêtes sont confidentielles. Les fonds collectés permettent de renflouer les caisses du trésor municipal et de financer de nombreux projets tels que la construction d'édifices scolaires, la fourniture en équipements de tables-bancs, l'entretien et la réhabilitation du réseau routier existant et l'ouverture de nouvelles voies.

Les entreprises exerçant sur l'espace portuaire constituent une source de création d'emplois. Les résultats des investigations en termes d'emplois générés par les entreprises installées à San-Pedro sont consignés dans le tableau 3.

Tableau 3: La distribution des employés par secteur d'activité à San-Pedro en 2016

\begin{tabular}{|l|c|c|c|c|c|c|}
\hline \multirow{2}{*}{ Secteur d'activité } & \multicolumn{7}{|c|}{ Nombre d'employés } \\
\cline { 2 - 7 } & ] 0-50] & ] $50-100]$ & ] 100-200] & ] $200-300]$ & ] 300 plus] & Total \\
\hline Cimenterie & 1 & 0 & 0 & 0 & 0 & 1 \\
\hline $\begin{array}{l}\text { Transformation de } \\
\text { bois }\end{array}$ & 0 & 3 & 3 & 2 & 4 & 12 \\
\hline Usine de cacao & 0 & 4 & 5 & 2 & 5 & 16 \\
\hline Société maritime & 3 & 4 & 2 & 1 & 0 & 10 \\
\hline Total général & 4 & 11 & 10 & 5 & 9 & 39 \\
\hline
\end{tabular}

Source : enquêtes de terrain, 2017

Au regard de ce tableau, le constat général est que toutes les entreprises disposent d'employés. En effet, sur 39 sociétés concernées par nos enquêtes 11 disposent de 50 à 100 employés. Il s'agit de 2 unités de de transformation de bois (SOFIBEX et WOOD IVORE), 4 usines de cacao (TOUTON, SUV, SAHN, MATRANCI) et 4 sociétés maritimes. Parmi les entreprises visitées, 10 ont un effectif compris entre 100 et 200 personnes, 9 emploient plus de 300 
personnes, 5 disposent de 200 à 300 employés et 4 emploient ont au plus 50 personnes. Ce sont la cimenterie SOCIM et trois sociétés maritimes (SOMITREX, ICM, AQUAMARINE). Ces chiffres attestent de l'importance des entreprises dans la création d'emplois. À côté des emplois générés, on note une prolifération d'activités informelles autour du port et dans la zone industrielle.

\section{2-3-Les zones portuaires et industrielles : foyers de diverses activités commerciales informelles génératrices de revenus}

L'existence des activités portuaires et industrielles a donné naissance à une multiplicité de commerces informels autour du port et dans la zone industrielle. Le commerce informel est dominé par les activités de vente de bois, de poissons, d'attiéké ${ }^{3}$ et les restaurants. Ces différentes activités constituent une importante source de revenu pour la population. Le tableau 4 ci-dessous donne quelques précisions relatives aux revenus générés par les activités informelles en rapport avec le port.

Tableau 4: Estimations de quelques revenus (F CFA) tirés du commerce informel

\begin{tabular}{|l|l|c|c|}
\hline Types d'activités & $\begin{array}{l}\text { Nombre de personnes } \\
\text { interrogées }\end{array}$ & $\begin{array}{l}\text { Revenu journalier moyen } \\
\text { de l'activité en F CFA }\end{array}$ & $\begin{array}{l}\text { Revenu mensuel en F } \\
\text { CFA }\end{array}$ \\
\hline Vente de poisson & 81 & 25000 & 750000 \\
\hline Vente d'attiéké & 35 & 8000 & 240000 \\
\hline Vente de bois & 37 & 15000 & 450000 \\
\hline $\begin{array}{l}\text { Vente de jus de } \\
\text { rafraîchissement }\end{array}$ & 12 & 2000 & 60000 \\
\hline Restauration & 31 & 12000 & 360000 \\
\hline Total & 196 & 62000 & 1860000 \\
\hline
\end{tabular}

Source : enquêtes de terrain, 2018

Le tableau 4 présente les revenus journaliers et mensuels de 196 personnes qui vivent au dépend du port sans que leurs activités ne soient liées directement au domaine maritime. L'analyse du tableau montre que les vendeuses de poissons ont le plus gros revenu. Il convient de souligner qu'un vendeur de poisson gagne environ $25000 \mathrm{~F}$ CFA par jour ce qui lui permet d'avoir 750000 F CFA en 30 jours d'activités. Cette somme est obtenue en multipliant le gain journalier par 30, c'est-à-dire le nombre de jours qu'il y a dans un mois. Les vendeurs de bois occupent la deuxième place avec un gain journalier qui tourne autour de $15000 \mathrm{~F}$ CFA. Ce qui donne un revenu mensuel de $450000 \mathrm{~F}$ CFA. En troisième et quatrième position viennent les restaurateurs (restauratrices) et les vendeurs (vendeuses) d'attiéké avec des recettes journalières respectives de 12000 et 8000 F CFA. Les vendeuses de

${ }^{3}$ Attiéké : Semoule de manioc 
jus de rafraichissement avec un revenu de $2000 \mathrm{~F}$ CFA par jour disposent du plus petit revenu.

Les emplois et les revenus occasionnés par la présence du port participent à la réduction de la pauvreté, à l'amélioration des conditions de vie des populations. Cependant, si l'ouverture du port a des retombées socioéconomiques importantes, il n'en demeure pas moins que les activités liées à l'existence du port ont des incidences négatives sur l'environnement.

\section{3-Les problèmes environnementaux induits par la présence du port à San- Pedro}

La ville de San-Pedro à l'instar des villes ivoiriennes est confrontée à des problèmes environnementaux d'origine diverses. Cette section met exergue les effets néfastes des activités portuaires sur l'environnement.

\section{3-1-Un paysage de lac ${ }^{4}$ pollué : conséquence des activités industrielles}

Le secteur industriel à San-Pedro est dominé par l'agro-industrie. Les agro-industries consomment beaucoup d'eaux et rejettent dans le lac d'importantes quantités d'eaux usées sans traitement. L'évacuation des eaux usées non traitées a des incidences négatives sur le lac. Elles ont fini par polluer cette manne naturelle. En effet, ce patrimoine naturel jadis convoité pour la pêche et qui constituait un atout touristique n'est plus reluisant. Il est de plus en plus envahi par des plantes aquatiques comme le montre la photo 1 .

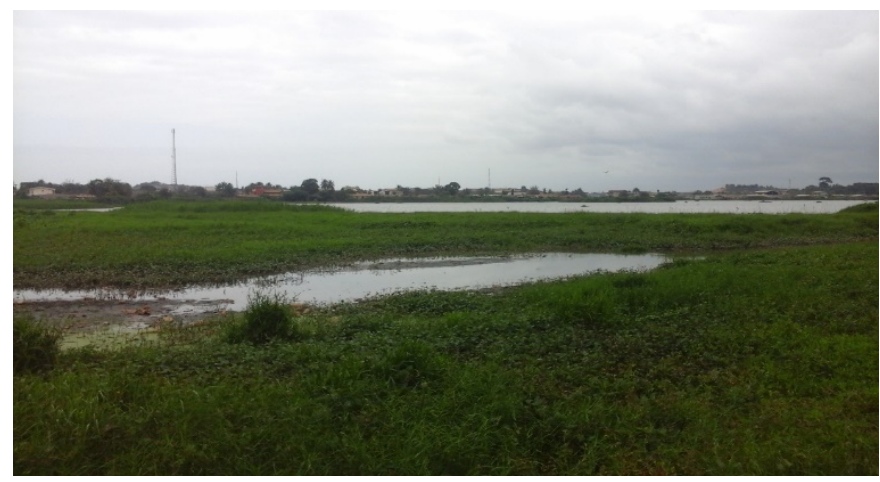

Photo 1 : Le lac de San-Pedro envahit par les plantes aquatiques Source : OGOU Atsé, 2017

La pollution du lac par les déchets industriels rend les eaux de ce lac impropres à toute activité économique. Cette situation a amené la direction régionale des eaux et forêts du Bas Sassandra à interdire la pratique de la pêche

${ }^{4}$ Cours d'eau naturel qui borde de nombreux quartiers de la ville de San-Pedro dont Manzn, zone industrielle, Jules Ferry... 
dans ce plan d'eau. La pollution de ce vaste environnement lacustre met en péril la vie de la faune qui s’y trouve.

À ces problèmes, s'ajoutent les nombreux remblaiements du lac par les opérateurs économiques pour la construction de magasins de stockage. L'installation des entreprises autour du lac s'explique, essentiellement, par l'absence de terrains industriels en zone portuaire et de l'opportunité que leur offre le plan d'eau lacustre. En effet, il est situé en bordure de la voie principale qui mène au port. Pour rendre plus dynamique leurs activités et réaliser des économies d'échelle, les opérateurs économiques ont choisi de s'installer aux abords du lac. Ils ont ainsi la possibilité d'acheminer plus facilement leurs marchandises vers le port. Ces actions contribuent davantage au rétrécissement progressif de cette étendue d'eau. Le service technique de la mairie indique que le lac se déployait sur environ huit ha dans les années 1960. Il ne s'étend plus que sur trois hectares aujourd'hui.

\section{3-2-Une physionomie actuelle de végétation dégradée avec des exploitations agricoles à perte de vue}

L'exploitation des ressources naturelles et agricoles pour l'alimentation du trafic portuaire a joué un rôle fondamental dans la prospérité dudit port. Cependant, la mise en valeur des richesses du Sud-ouest a des effets contraignants sur le milieu naturel. Elle a engendré de profondes transformations du paysage végétal. Aujourd'hui, la situation du secteur forestier dans la région du Sud-ouest et particulièrement dans le département à San-Pedro est extrêmement préoccupante (figure 3).

Figure 3: Structure actuelle du couvert forestier du département de San-Pedro

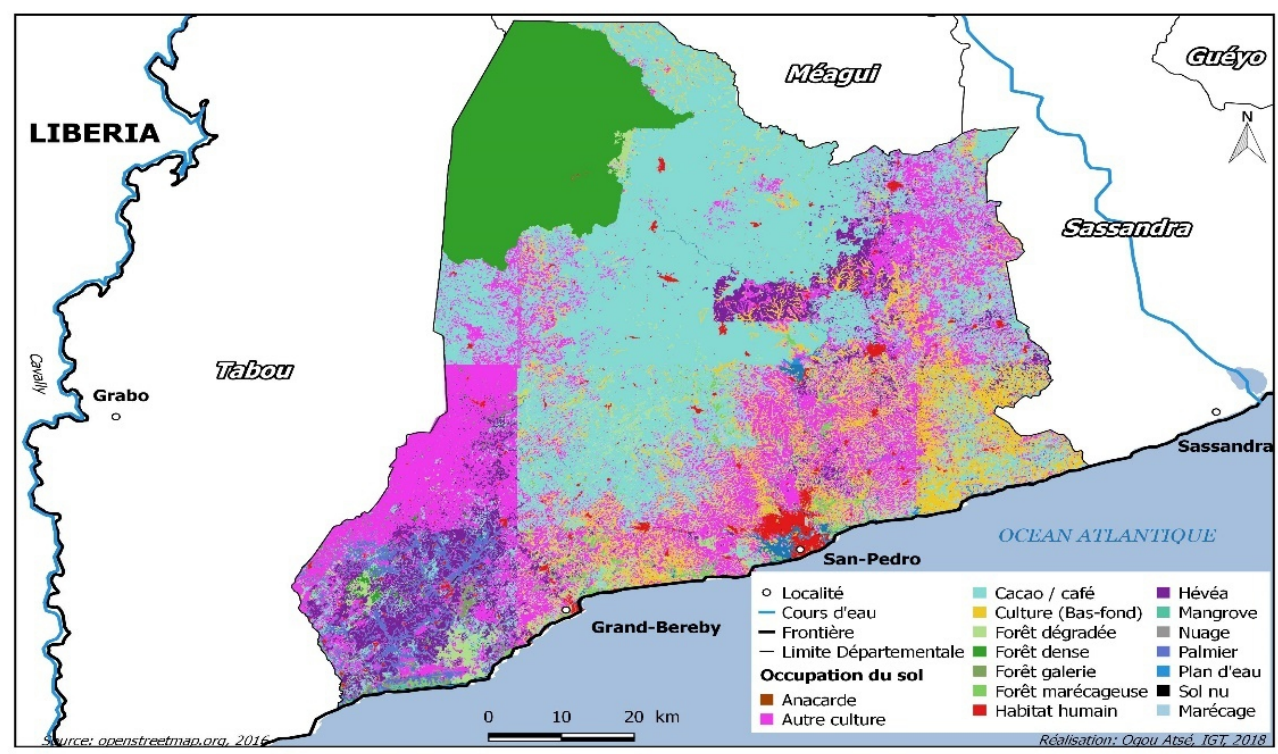


L'exploitation forestière et les activités agricoles constituent les principales sources du recul du massif forestier. Le premier élément cité a joué le rôle précurseur et le second le facteur amplificateur de la disparition des surfaces de forêts (figure 4).

Figure 4: Les différentes phases de transformation du milieu forestier du Sud-ouest ivoirien

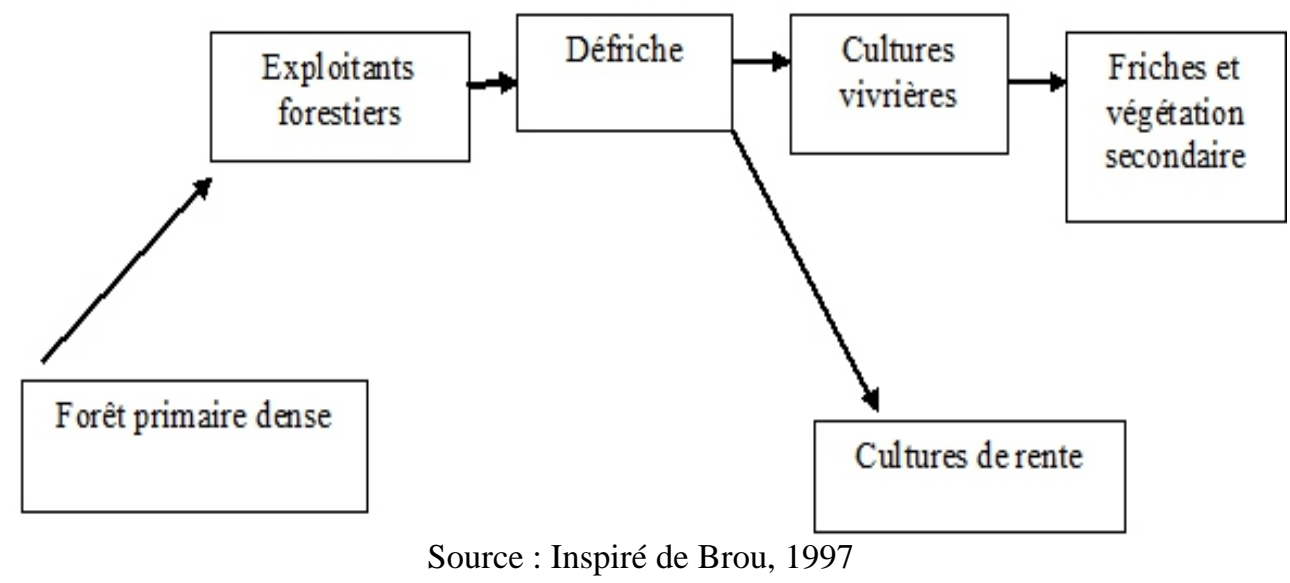

En effet, les exploitants forestiers infiltrent en premier les forêts luxuriantes regorgeant d'innombrables essences. Ils prélèvent les ressources ligneuses à l'aide de tronçonneuses, de bulldozers et acheminent, les grumes avec des camions remorques (grumiers) vers les parcs des unités industrielles de la ville de San-Pedro pour une semi-transformation ou directement vers le port.

Les populations paysannes suivent les pistes créées par les exploitants forestiers en réalisant des plantations villageoises de café et de cacao principalement à travers un système d'exploitation basé sur des méthodes culturales extensives sur brûlis. La conquête des terres s'est accentuée avec l'arrivée des blocs agro-industriels $\left(\mathrm{SICOR}^{5}, \mathrm{SOGB}^{6}, \mathrm{PALMCI}^{7}\right.$, SIPEFCI ${ }^{8} \ldots$ ) qui créent de vastes plantations industrielles (palmier à huile, hévéa, cocotier) s'étendant sur des dizaines, voire des centaines d'hectares. Les productions agricoles issues des plantations villageoises et industrielles sont profitables au port en ce sens qu'elles permettent à ce dernier de diversifier son trafic composé autrefois essentiellement d'exportation de grumes.

Le défrichement abusif et irrationnel de la forêt à des fins de production agricole a entrainé la rapide disparition de la quasi-totalité du manteau forestier. Selon Léonard et Vimard (2005, p. 18), ce sont 2 millions

${ }^{5}$ SICOR : Société Ivoirienne de Coco Rapé

${ }^{6}$ SOGB : Société des Caoutchoucs de Grand-Béréby

${ }^{7}$ PALMCI : Palm Côte d'Ivoire

${ }^{8}$ SIPEFCI : Société Internationale de Plantations et de Finance en Côte d'Ivoire 
d'hectares (80 \% de la surface totale) qui ont été défrichés en 20 ans dans le Sud-ouest ivoirien. Par ailleurs, la région présente à ce jour un paysage composé en majorité de lambeaux de forêts et d'immenses plantations de cultures de rente (cacao, café, palmier à huile et hévéa). La régression des ressources forestières face aux besoins de nouvelles terres arables et d'extension des superficies exploitables a poussé les populations paysannes à infiltrer de façon clandestine les forêts classées pour la pratique des activités agricoles. Cette situation a occasionné la disparition progressive des surfaces des forêts classées. La SODEFOR estime que 70 \% des 640000 ha de forêts classées du Sud-ouest de la Côte d'Ivoire sont dégradées (SRAT, 2008, p. 41).

La disparition du couvert forestier expose le département de San-Pedro à de nombreux problèmes. Ce sont entre autres l'appauvrissement des sols, la multiplication des conflits fonciers, les perturbations climatiques et la fermeture de certaines scieries. Cette situation constitue une menace pour la continuité des activités portuaires, d’autant plus que la pénurie de ces ressources va entraîner la baisse du trafic maritime. Ce qui va engendrer une léthargie dans le développement économique du département de San-Pedro.

\section{3-3-L'érosion côtière : un phénomène accentué par le développement des ouvrages portuaires}

Le littoral de San-Pedro avec la construction du port dans les 1970 a fait l'objet de nombreux travaux d'aménagement. Les principales interventions ont porté sur la réalisation des jetées Ouest et Est, le détournement vers l'Est de la rivière San-Pedro, la mise en place de dispositif d'arrêt de sables et plusieurs travaux de dragage. Ces ouvrages perturbent le transit sédimentaire, entrainant l'instabilité des plages situées entre la jetée Est et le club nautique. Cette partie du littoral subit la conséquence, de façon marquante, de l'interruption du transit sédimentaire Ouest-Est, mais également de la configuration de la côte et de l'énergie du milieu (Yao et al, 2010, p. 5).

Par conséquent, les activités économiques, les installations hôtelières touristiques et les arbres situés à proximité de ce rivage sont soumis à l'érosion (photo 2). Ce secteur a enregistré des vitesses moyennes de recul de l'ordre de $1.2 \mathrm{~m} / \mathrm{an}$ au cours de la période 1993-1997 (Yao et al, 2010, p. 7). 


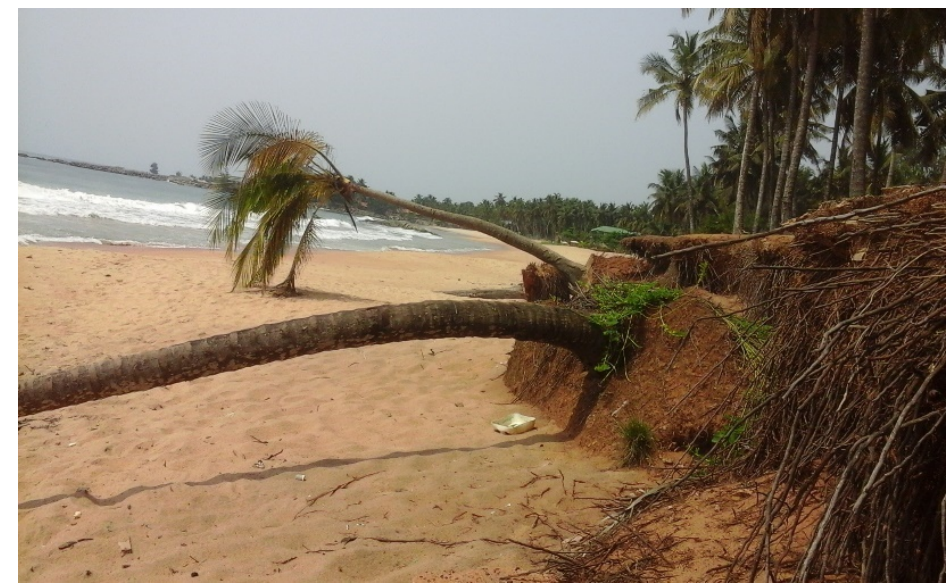

Photo 2: Des cocotiers inclinés sous l’action de l'érosion

Source : OGOU Atsé, 2017

\section{3-4-Lepoids déterminant des industries dans la pollution atmosphérique et sonore}

Les activités issues des entreprises industrielles sont des facteurs importants de pollution atmosphérique. En effet, elles rejettent des poussières et de nombreux gaz comme le dioxyde de carbone, le méthane, etc. (photo 3) qui se propage dans l'atmosphère sous l'action du vent. Ces gaz ou poussières sont dangereux pour l'environnement.

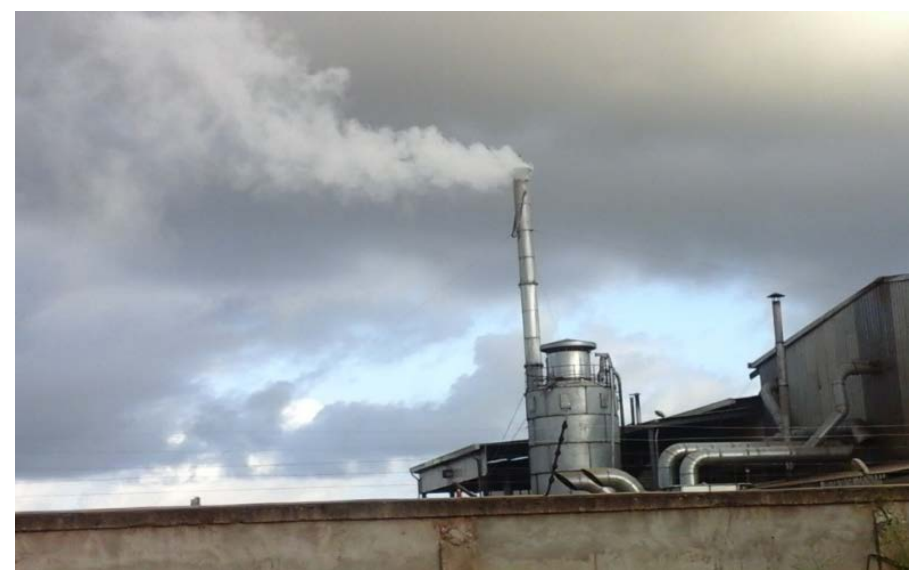

Photo 3: Émission de fumée par une usine de transformation de cacao à San-Pedro Source : OGOU Atsé, 2017

Outre l'impact négatif des polluants sur la qualité de l'air, ils exposent les populations riveraines et les travailleurs de la zone industrielle à des nuisances et infections. Les populations des quartiers Bardo, Séwéké et Zimbawé en raison de leur situation géographique (proche de la zone industrielle) sont les plus vulnérables. Elles sont exposées aux maladies respiratoires comme les bronchites, les asthmes, les sinusites, etc. 
À ces désagréments, s’ajoutent les nuisances sonores causées par le bruit des machines. Les problèmes susmentionnés exposent la ville de San-Pedro et toute la région du Sud-ouest à de nombreux risques.

\section{3-4- Une ville exposée à de multiples risques}

La ville de San-Pedro est exposée à deux types de risque : industriel et écologique.

3-4-1-Les risques industriels

Les risques industriels sont définis comme étant une combinaison de l'aléa et de la vulnérabilité (Zohr, 2014, p. 201). San-Pedro du fait de la présence des industries de bois et agro-alimentaires n'est pas à l'abri d'éventuelles catastrophes. L'enquête de terrain a permis de relever deux types de risques. Il s'agit de l'incendie et de l'explosion. Le risque d'incendie résulte en grande partie du contact entre les étincelles ou les particules incandescentes avec un corps inflammable. Il peut être également engendré par des courtscircuits dus aux pannes électriques. Il a été constaté dans certaines usines de bois des fils électriques découverts qui se touchent. Ils peuvent engendrer un incendie. Le risque d'explosion est plus présent dans les industries chimiques et agro-alimentaires. En effet, elles utilisent le gaz comme source d'énergie. De ce fait, un dysfonctionnement du système d'alimentation (fuite de gaz) peut entraîner des explosions aux conséquences désastreuses.

La vulnérabilité de la ville de San-Pedro face à ces menaces risque de s'accroître avec l'exécution du projet d'extension du port qui prévoit la construction et l'exploitation d'un dépôt d'hydrocarbure et d'un quai pétrolier. La mise en œuvre de ce projet de grande envergure représente une opportunité pour les industriels d'autant plus qu'il va engendrer l'implantation de nombreuses industries spécialisés dans le traitement de produits pétroliers. Ces installations sont à l'origine de divers risques environnementaux. L'incendie déclenché dans la Société de Gestion des Stocks Pétroliers de Côte d'Ivoire (GESTOCI) en 1999 et celui survenu en Janvier 2017 à la Société Ivoirienne de Raffinage (SIR) témoigne de l'existence de risque d'explosion dans la zone industrialo-portuaire à Abidjan. Dans les faits, ces évènements ont entraîné d'importants dégâts matériels et la pollution de l'air aux alentours de la zone industrielle de Vridi (Abidjan). Ces constats permettent de déduire que l'installation la construction et l'exploitation d'un dépôt d'hydrocarbure et d'un quai pétrolier au port de San-Pedro expose la population à de réels dangers.

3-4-2-La disparition du couvert forestier, source de divers problèmes

La destruction des forêts a des incidences socio-économiques et écologiques qui se manifestent par la perturbation du climat, l'appauvrissement des sols, les risques de fermeture des scieries et la propagation de conflits fonciers. 
Dans la région de San-Pedro, la disparition de la grande partie du couvert forestier commence à avoir des incidences sur les éléments climatiques. À ce propos, l'étude monographique et économique réalisé par le Ministère d'État, Ministère du plan et du développement (MEMPD) en 2014, à partir de l'analyse des hauteurs pluviométrique entre 1971 et 2000 indique que la région du Sud-ouest enregistre de fortes perturbations climatiques. La même source stipule que la pluviométrie a diminué d'environ $200 \mathrm{~m}$. L'appauvrissement des sols fait partie intégrante des effets néfastes de la déforestation sur l'environnement. La forêt et les sols entretiennent des relations étroites. Brou et Chaléard (2007, p. 67) révèlent que la forêt dense humide à travers l'épaisseur de sa végétation et ses racines exercent sur les sols un rôle protecteur. Roose (1984, p. 26) souligne à cet effet que le défrichement des terres à des fins agricoles favorise la détérioration des propriétés chimiques et physiques des horizons superficiels du sol. Pour ce faire, la dynamique des activités forestières et agricoles que connaît la région de San-Pedro menace la fertilité des sols. L'irrégularité pluviométrique combinée à l'appauvrissement des sols peut conduire à la baisse des productions agricoles. Cette situation peut mettre en péril dans les années futures la sécurité alimentaire d'une frange importante de la population tant en milieu rural qu'urbain.

À ces problèmes, s'ajoute la régression des terres cultivables due en partie à l'agriculture extensive et basée sur la technique de culture itinérante sur brûlis. La saturation foncière est à l'origine de nombreux conflits fonciers dans la région Sud-ouest. Les litiges identifiés peuvent être regroupés en quatre catégories. On distingue : les conflits opposant les autochtones aux migrants (allochtones et allogènes) ; les allochtones aux allogènes ; les autochtones entre eux et l'État ivoirien contre les clandestins (occupants des réserves et parcs nationaux). Ces différentes formes de conflits se développent majoritairement dans les zones très peuplées et dans les zones de grandes productions cacaoyères et caféières (SRAT, 2008, p 45). Toujours selon la même source, les zones les plus conflictuelles sont les départements de SanPedro et Soubré, avec respectivement $57 \%$ et $25 \%$ des conflits enregistrés. Ces deux départements sont les plus peuplés et les plus attractifs de la région de par leur situation géographique, leur importance économique et leurs poids démographique. Ils regroupent, à eux seuls, $75 \%$ de la population totale de la région. La persistance des conflits risque à la longue d'être un obstacle majeur au développement de l'agriculture indispensable au développement des activités portuaires.

La déforestation a également entrainé une pénurie de bois dans l'arrière-pays national du port de San-Pedro. De ce fait, les essences très prisées comme l'acajou, le Makoré, le Tiama et l'Aniegré qui dominaient dans la région se sont raréfiés. Ce qui contraint les sociétés de bois à se tourner vers 
les essences comme (fromager, samba, ako, etc.) autrefois ignorées pour leur qualité jugée inférieur. Celles-ci commencent également à se faire de plus en plus rares.

Comme solutions palliatives à la pénurie de la ressource ligneuse, les grands groupes tels que Thanry, Stbs, African Industrie... ont étendu leurs tentacules aux pays limitrophes (la Guinée forestière et le Libéria) où il existe encore d'importantes réserves de bois d'usine pour se procurer la matière première. Ces entreprises reçoivent dans ces pays des autorisations temporaires d'importation de grumes. Ce fut le cas pour African Industrie en 2006. Les scieries de tailles moyennes comme Wood Ivoire et Sofibex arrivent un tant soit peu à satisfaire leur besoin grâce aux essences fournies par la SODEFOR. Les espèces livrées sont constituées majoritairement de Cédrela et de Teck. La tendance baissière en fourniture de grumes menace l'avenir de la filière bois à San-Pedro d'autant plus qu'elle va entraîner la fermeture de plusieurs unités industrielles. La photo 4 présente le parc à bois de la société Wood Ivoire presque vide de ressources ligneuses.

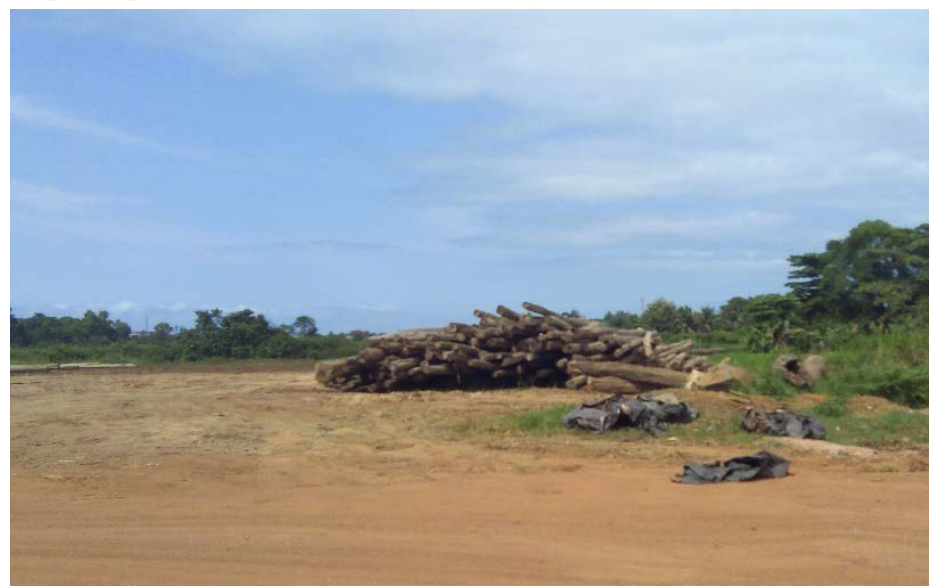

Photo 4: Parc à bois désert de la scierie Wood Ivoire Source: OGOU Atsé, 2017

Selon le chef de parc de cette unité industrielle, le parc à bois était continuellement rempli d'essences ligneuses. Cela montre combien de fois la rareté de bois affecte les acteurs de la filière bois au niveau de productivité. La disparition des scieries aura des conséquences néfastes pour les travailleurs dans la mesure où elle va occasionner de nombreux licenciements. Ce qui va accroître le taux de chômage. Ceci constitue une véritable perte car une unité de transformation de bois en emplois en moyenne 100 personnes.

\section{DISCUSSION}

Cette étude a permis de suivre les différents investissements réalisés pour le développement du port de San Pedro et le Sud-ouest ivoirien. Il découle des 
investigations que les activités en rapport avec le port se font au détriment des principes du développement durable. La croissance des trafics du port a reposé principalement sur la mise en valeur des ressources naturelles considérées comme "inépuisables". Cette politique a provoqué une surexploitation de la ressource ligneuse. Aussi faut-il noter le caractère fortement extensif de l'agriculture basée sur la technique de culture itinérante sur brûlis. Ce type d'agriculture a contribué en grande partie à une saturation foncière. Elle expose la région du Sud-ouest à des risques écologiques. On note par ailleurs une gestion des déchets industriels très peu respectueuse de l'environnement. Ces résultats sont identiques à ceux de De Raymond (2003, p. 61-62). L'auteur montre que les nombreux aménagements effectués sur l'estuaire de la Loire (France) ont d'une part engendré une forte croissance des trafics portuaires et d'autre part dégradé l'estuaire et pénalisé les autres usages économiques (agriculture, tourisme pêche, etc.). Les travaux de Zohra (2014, p. 189- 202) ont abouti à cette même conclusion. Elle démontre que dans la ville portuaire de Skikda (Algérie), le développement de l'activité industrielle se fait aux dépens de l'environnement. Elle souligne à cet effet que Skikda est vulnérable aux risques industriels tels que les incendies et les explosions. Les résultats de la présente étude sont également en harmonie avec les travaux de N'doufou et al (2015, p. 2-8). Les auteurs indiquent que l'ouverture du canal de Vridi et les ouvrages de protection qui lui sont associés font barrage à la dérive littorale, provoquant un engraissement général à l'Ouest et une érosion à l'Est. Comme conséquence immédiate, on assiste à l'érosion de la baie de PortBouët. Contrairement à ces études, celles de Labaronne (2014, p. 177-197) montrent que le déploiement des activités portuaires dans la ville de Béjaïa (Algérie) est compatible avec les exigences du développement durable. L'auteur révèle que cet état de fait est à mettre à l'actif à l'Entreprise portuaire de Béjaïa (EPB). Les responsables de cette structure ont mis en place une politique de Responsabilité Sociale des entreprises (RSE). Ce qui permet de concilier performances économiques portuaires, contraintes environnementales et développement durable urbain.

Dans le Sud-ivoirien, la régression des milieux naturels selon Adayé (2013, p. 300) s'explique par la forte pression foncière et les conditions d'exploitation forestière non conformes aux règles d'exploitation durable (très faible niveau de reboisement, présence d'opérateurs peu professionnels qui exploitent la forêt comme une rente dans une logique de profit à court terme). Tapé (2011, p. 411- 412) impute la disparition des forêts aux entreprises agroindustrielles dont les plantations occupent des milliers d'hectares. Face à la dégradation de l'environnement, de nombreuses actions ont été entreprises par les structures techniques. Cependant, ces initiatives présentent des limites. L’inefficacité des actions se justifie par le manque de moyens matériels et humains des structures en charges de l'environnement. Ces facteurs limitent 
les actions de terrain entreprises par les organes étatiques régionales pour protéger durablement l'environnement. (Kassi ,2012 ; p. 213-214) abonde dans ce sens. Il avance que l'échec des stratégies est dû au manque de synergie entre les industriels et les structures étatiques en charge de l'environnement.

\section{CONCLUSION}

San-Pedro doit son développement actuel à son port. L'ouverture de cette infrastructure économique a engendré l'installation d'une multiplicité d'entreprises exerçant pour la plupart dans l'agro-alimentaire, la transformation de bois et les services à la marchandise exécutés par les sociétés maritimes. Leur présence a été d'une importance cruciale dans la dynamique des activités portuaires et par ricochet le développement de l'espace urbain de San-Pedro. En effet, cette localité qualifiée autrefois de désert humain est devenue par le biais du port et les sociétés qui l'accompagnent une ville attractive. Elle représente aujourd'hui le deuxième pôle économique de la Côte d'Ivoire après Abidjan. Les diverses activités qui se déroulent dans la zone portuaire et industrielle génèrent des revenus et de nombreux emplois aux populations. Ce qui permet aux travailleurs de se prendre en charge et de subvenir aux besoins de leur famille.

Cependant, derrière ces progrès sociaux et économiques se cachent des problèmes environnementaux qui se matérialisent par une disparition rapide du couvert forestier, l'érosion côtière et des pollutions diverses. Les pollutions sont dues en majorité à la mauvaise gestion des déchets industriels. Elles se traduisent par la dégradation de la qualité de l'air, des eaux du lac de SanPedro. Les pollutions exposent la population à de nombreuses maladies. Quant à la destruction des forêts, elle menace les sols, le climat et la fermeture des scieries. Face aux problèmes énumérés, il serait intéressant que les structures en charge de l'environnement s'engage résolument à l'application effective du code de l'environnement.

\section{References:}

1. Adaye A. A. (2013). Développement agricole et sécurité alimentaire dans la région du Bas- Sassandra (Sud-ouest de la Côte d'Ivoire), thèse Unique de Doctorat, IGT, Université Félix Houphouët-Boigny, Abidjan, 512 p.

2. Brou Y. (1997). Le Sud forestier et sa transformation par les activités humaines, Analyse et dynamique de la pluviométrie dans le Sud forestier ivoirien: recherche de corrélation entre les variables climatiques et les variables liées aux activités anthropiques, Thèse de $3^{\mathrm{e}}$ cycle en Géographie, Abidjan, ORSTOM, p 118-187. 
3. Brou Y. et Chaleard J-L. (2007). «Visions paysannes et changements environnementaux en Côte d'Ivoire ", in Annales de géographie, $n^{\circ}$ 653, Armand Colin, Paris, p 65-87.

4. Brundtland. (1987), Rapport Brundtland, notre avenir à tous, p 38-45.

5. Choblet C. (2005). Espace littoral et décisions d'aménagement, Limites et potentialités des études d'impact et des enquêtes publiques. Exemple du littoral atlantique français, thèse de doctorat, Université de Nante, p 50-57.

6. De Raymond T. (2003). « Concilier développement portuaire et autres usages d'un estuaire : le cas de la Loire ", in Collin M (dir). Ville portuaire, acteur du développement durable, l'harmattan, p 61-66.

7. Hauhouot A. A. (2002). Développement, aménagement, régionalisation en Côte d'Ivoire, Abidjan, EDUCI, 371 p.

8. Kablan N. H. J.( 2008). «Importance des ports dans l'économie ivoirienne », in ANOH (K .P.) et POTIER (P.) (dirs). Géographie du littoral de Côte d'Ivoire, éléments de réflexion pour une politique de gestion intégré, p 83-102.

9. Kablan N.H J et Kouakou N N. (2013). « Impact environnemental de la zone industrialo-portuaire (zip) d'Abidjan » in European Scientific Journal, n 35 vol 9, p 206-221.

10. Kassi K .J. C. (2012) Les entreprises industrielles et la gestion de l'environnement dans la ville de San-Pedro, thèse de Doctorat, université Felix Houphouët Boigny, Abidjan, 264 p.

11. Labaronne D. (2014). «Responsabilité sociale des entreprises et développement durable des villes portuaires: cas d'une entreprise publique portuaire algérienne " in LABARONE (Coord). Villes portuaires au Maghreb : acteurs du développement durable, presses des Mines, collection Développement durable , p 176-197.

12. Léonard E, Vimard P. (2005). Crises et recompositions d'une agriculture pionnière en Côte d'Ivoire : Dynamiques démographiques et changements économiques dans le Bas-Sassandra, IRD et Karthala, p 17-20.

13. Loba A. D. V. (2010). «La ville de San-Pedro en Côte d'Ivoire itinéraire de développement d'un pôle régional côtier » in $R G L L, \mathrm{n}^{\circ} 08$ $22 \mathrm{p}$.

14. Ministère d'État, Ministère du Plan et du Développement (MEMPD), Direction Générale du Développement de l'Économie Régionale (2008). Schéma Régional d'Aménagement du Territoire du BasSassandra (SRAT), Côte d'Ivoire, Abidjan, 112 p.

15. Ministère d'État, Ministère du Plan et du Développement (MEMPD), 2015, Études monographiques et économiques de Côte d'Ivoire : District du Bas Sassandra, 343 p. 
16. N’doufou G, Abe J, Bamba S, Hauhouot C, Aka K. (2015). « Effets de l’ouverture du Canal de Vridi sur les stocks sédimentaires littoraux entre Abidjan et Jacqueville (Côte d'Ivoire) », in Paralia, Vol. 8, p 116.

17. N’guessan A.A. B., 2010. L'avant-pays européen du port de SanPedro, thèse unique de doctorat, université de Cocody, Abidjan, 465p.

18. N’guessan A. B. (2013). « Port et aménagement du territoire en Côte d'Ivoire : bilan et perspectives, à partir de San-Pedro » in $R G O \mathrm{n}^{\circ} 002$, p 123-140.

19. N’guessan A.B.et Loba A.V. (2015), « Ports et aménagements urbains dans les villes portuaires », in N. ESCACH, (dir.), Géographie des mers et des océans, Dunod, Paris-France, p. 292-326.

20. N’guessan A.B.et Loba A.V. (2017). Le port de San-Pedro, moteur du développement urbain et régional dans le Sud-ouest ivoirien, In Loba (A. D) et N'guessan (A. B) (dirs). Le port dans la ville, France, EMS Edition, p 103-126.

21. Roose E., 1984. « Impact du défrichement sur la dégradation des sols tropicaux. Machinisme Agricole Tropical », in ORSTOM, n $8724-36^{\mathrm{e}}$ réunion technique 55ème S I M A p 24-36.

22. Schwartz A. (1970). Recensement démographique de la Souspréfecture de San-Pedro, vol 3, Abidjan, 80 p.

23. Schwartz A. (1993). Sous-peuplement et développement dans le Sudouest de la Côte d'Ivoire, Orstom, Col. Etudes et thèse, Paris 490 p.

24. Tapé B. J. (2004). Économie maritime et portuaire de Côte d'Ivoire : étude géographique, thèse de doctorat d’État, université d'Abidjan, IGT, 876 p.

25. Tapé S P. (2011). Aménagement et gestion des villes côtières du BasSassandra : cas de Tabou, Grand-Bereby, San-Pedro et Sassandra, Thèse Unique de Doctorat, IGT, Université Félix Houphouët-Boigny, Abidjan 540 p.

26. Yao K.S., ABE J., BAMBA S.B., KONAN K.E., AKA K. (2010). « Dynamique d'un périmètre littoral portuaire : la côte de San-Pedro, Sud-ouest de la Côte d'Ivoire » in Paralia, Vol. 3, p1-12.

27. Zohra M-C F. (2014). « Port, risques industriels et développement durable des territoires : la vulnérabilité de la ville portuaire de Sikda (Algerie) » in LABARONE (Coord). Villes portuaires au Maghreb : acteurs du développement durable, presses des Mines, collection Développement durable p 199-211. 